\title{
Frequency-Domain Multiuser Detection for CP-Assisted DS-CDMA Signals
}

\author{
Paulo Silva and Rui Dinis \\ ISR-IST, Tech. Univ. of Lisbon, Portugal, \{psilva, rdinis\}@ist.utl.pt
}

\begin{abstract}
In this paper we consider the use of CPassisted (Cyclic Prefix) DS-CDMA schemes (Direct Sequence Code Division Multiple Access) in broadband wireless systems. We present an iterative, frequency-domain MUD (MultiUser Detection) receiver for the uplink transmission that combines IB-DFE (Iterative Block Decision Feedback Equalization) principles with serial interference cancelation.

It is shown that the performance of the proposed receiver can be close to the single-user MFB (Matched Filter Bound), even for fully loaded systems, in severely time-dispersive channels and/or in the presence of strong interfering signals. ${ }^{1}$
\end{abstract}

\section{Introduction}

When employed in cellular systems, DS-CDMA schemes (Direct Sequence Code Division Multiple Access) [1] allow good capacities, together with high system flexibility. Moreover, contrarily to TDMA schemes (Time Division Multiple Access), all users transmit continuously, regardless of the bit rates, reducing significantly the peak power requirements for the amplifiers. This, combined with the relatively low envelope fluctuations of the DS-CDMA signal associated to each spreading sequence, makes DS-CDMA schemes good candidates for broadband wireless systems, especially at the uplink (i.e., the transmission form the MT (Mobile Terminal) to the BS (Base Station)).

However, for severe time-dispersive channels the loss of orthogonality between users can lead to significant performance degradation, unless very high-complexity MUD (MultiUser Detection) receiver structures are employed.

Block transmission techniques, with appropriate $\mathrm{CP}$ (Cyclic Prefix) and employing FDE (Frequency-Domain Equalization), are suitable for high data rate transmission over severe timedispersive channels, since they allow low-complexity, FFTbased (Fast Fourier Transform) implementations [2], [3].

A CP-assisted OFDMA (Orthogonal Frequency Division Multiple Access) scheme was considered in [4] for the uplink of broadband wireless systems, where all users transmit continuously a signal with reduced envelope fluctuations [5][7]. As with conventional OFDMA schemes, the different users remain orthogonal on time-dispersive channels, simplifying the receiver design. However, a good carrier synchronization

\footnotetext{
${ }^{1}$ This work was partially supported by the FCT project POSI/CPS/46701/2002 - MC-CDMA and the B-BONE project IST2003-507607.
}

between users is required. Moreover, since just a fraction of the available subcarriers is assigned to each user, the achievable performances are worse than the corresponding SC schemes [4].

As an alternative, we can combine DS-CDMA schemes with $\mathrm{CP}$-assisted block transmission techniques, allowing a frequency-domain receiver design that have relatively low complexity, even for severely time-dispersive channels. The receiver is particularly simple at the downlink: since all spreading codes are affected by the same multipath channel, the receiver can be based on a simple FDE, operating at the chip level, followed by the despreading procedure [8], [9] .

To avoid significant noise enhancement, the FDE is usually optimized under the MMSE (Minimum Mean-Squared Error) criterion [10]. Since an MMSE FDE does not perform an ideal channel inversion, when this type of equalizer is employed in DS-CDMA systems we are not able to fully orthogonalize the different spreading codes. This means that we can have significant residual interference levels when different users have different powers. To avoid this problem, a promising nonlinear receiver structure was proposed in [11] which employs an IBDFE (Iterative Block Decision feedback Equalization) [12] especially designed for DS-CDMA signals. The IB-DFE can be regarded as a blockwise DFE where the feedforward and feedback parts are implemented in the frequency domain. Since the feedforward and feedback coefficients take into account the blockwise reliability, we have a turbo-like behavior, with small error propagation (in fact, the IB-DFE can be regarded as a low-complexity turbo equalization scheme [13], since the feedback loop does not use the channel decoder output).

In this paper we consider the uplink transmission within a DS-CDMA system employing CP-assisted block transmission techniques. We present an iterative frequency-domain MUD receiver that combines IB-DFE principles with MAI (Multiple Access Interference) cancelation techniques.

This paper is organized as follows: the CP-aided block transmission DS-CDMA schemes considered here are described in sec. II. In sec. III we describe the principles behind frequencydomain detection of CP-assisted DS-CDMA signals. In sec. IV we describe the iterative MUD receiver proposed in this paper. Sec. V presents a set of performance results and sec. VI is concerned with the conclusions of the paper.

\section{Block Transmission DS-CDMA}

In this paper we consider the uplink transmission in DSCDMA systems employing CP-assisted block transmission 
techniques. We have $P$ users and it is assumed that the blocks transmitted by each user have the same dimensions and there is a suitable "time-advance" mechanism allowing perfect synchronization in time at the receiver (in practice, just a coarse synchronization is required since some time misalignments can be absorbed by the CP). For the sake of simplicity, it is assumed that all users have the same spreading factor $K$ and the same data rate; however, these techniques can be easily extended to both VSF schemes (Variable Spreading Factor) and to multicode schemes [14].

The size- $M$ data block to be transmitted by the $p$ th user is $\left\{a_{n, p} ; n=0,1, \ldots, M-1\right\}$, with $a_{n, p}$ selected from a given constellation. The corresponding chip block to be transmitted is $\left\{s_{n, p} ; n=0,1, \ldots, N-1\right\}$, where $N=M K$ and $s_{n, p}=a_{\lfloor n / K\rfloor, p} c_{n, p}(\lfloor x\rfloor$ denotes "larger integer not higher than $x ")$, with $c_{n, p}$ denoting the spreading symbols. Throughout this paper it is assumed that $c_{n, p}= \pm \sqrt{2} / 2 \pm$ $j / \sqrt{2} / 2, n=m K, m K+1, \ldots, m K+K-1$, is the product of an $K$-length Hadamard-Walsh sequence with a pseudo-random QPSK sequence (Quadrature Phase Shift Keying), common to all users of the BS; the spreading sequence is also assumed to be periodic, with period $K$ (i.e., $c_{n+K, p}=c_{n, p}$ ).

The signal received at the BS is sampled at the chip rate (the generalization for multiple samples per chip is straightforward) and the $\mathrm{CP}$ is removed, leading to the time-domain block $\left\{y_{n} ; n=0,1, \ldots, N-1\right\}$. It can be shown that, when the $\mathrm{CP}$ is longer than the overall channel impulse response for each user, the corresponding frequency-domain block is $\left\{Y_{k} ; k=0,1, \ldots, N-1\right\}$, where

$$
Y_{k}=\sum_{p=1}^{P} S_{k, p} \xi_{p} H_{k, p}^{C h}+N_{k}
$$

with $H_{k, p}^{C h}$ denoting the channel frequency response for the $p$ th user and the $k$ th frequency and $N_{k}$ the channel noise for that frequency. $\xi_{p}$ is a scale factor accounting for the overall attenuation between the $p$ th MT and the BS. The frequency-domain block $\left\{S_{k, p} ; k=0,1, \ldots, N-1\right\}$ is the DFT of the chip block transmitted by the $p$ th user, $\left\{s_{n, p} ; n=\right.$ $0,1, \ldots, N-1\}$. Since $s_{n, p}=a_{\lfloor n / K\rfloor, p} c_{n, p}$, it can easily be shown that

$$
S_{k, p}=A_{k, p}^{\prime} C_{k, p}^{\prime}
$$

where $\left\{A_{k, p}^{\prime} ; k=0,1, \ldots, N-1\right\}=$ DFT $\left\{a_{n, p}^{\prime} ; n=\right.$ $0,1, \ldots, N-1\}$, with

$$
a_{n, p}^{\prime}= \begin{cases}a_{n^{\prime}, p}, & n=n^{\prime} K, \\ 0, & \text { otherwise }\end{cases}
$$

and $\left\{C_{k, p}^{\prime} ; k=0,1, \ldots, N-1\right\}=$ DFT $\left\{c_{n, p}^{\prime} ; n=\right.$ $0,1, \ldots, N-1\}$, with

$$
c_{n, p}^{\prime}= \begin{cases}c_{n, p}, & 0 \leq n<K \\ 0 . & \text { otherwise. }\end{cases}
$$

Clearly, $A_{k, p}^{\prime}=\frac{1}{K} A_{k \bmod M, p}, k=0,1, \ldots, N-1$, with $\left\{A_{k, p} ; k=0,1, \ldots, M-1\right\}=$ DFT $\left\{a_{n, p} ; n=0,1, \ldots, M-\right.$ $1\}$. This means that, apart a constant, the block $\left\{A_{k, p}^{\prime} ; k=\right.$
$0,1, \ldots, N-1\}$ is the size- $N$ periodic extension of the DFT of the data block associated to the $p$ th user $\left\{A_{k, p} ; k=\right.$ $0,1, \ldots, M-1\})$. This multiplicity in the $A_{k, p}^{\prime}$ is related to the spectral correlations that are inherent to the cyclostationary nature of the transmitted signals [15]. Therefore,

$$
Y_{k}=\sum_{p=1}^{P} A_{k, p} H_{k, p}+N_{k}
$$

with $H_{k, p}=\frac{1}{K} \xi_{p} H_{k, p}^{C h} C_{k, p}^{\prime}$ denoting the equivalent channel frequency response for the $p$ th user and the $k$ th frequency.

\section{Frequency-Domain Detection of CP-Assisted DS-CDMA Signals}

\section{A. Single-User Scenario}

1) Linear Receiver: Let us first consider the detection of the user 1 in a single-user scenario (i.e., $p=P=1$ ). The received frequency-domain samples are given by $Y_{k}=A_{k, 1} H_{k, 1}+N_{k}$. Since $A_{k+l M, 1}^{\prime}=A_{k, 1}^{\prime}=A_{k, 1} / K, l=0,1, \ldots, K-1$, there is a $K$-order implicit diversity effect in $Y_{k}$ and the detection could be made from

$$
\tilde{A}_{k, 1}=\sum_{l=0}^{K-1} Y_{k+l M} F_{k+l M, 1}
$$

where

$$
F_{k+l M, 1}=\frac{H_{k+l M, 1}^{*}}{\alpha_{1}+\sum_{l=0}^{K-1}\left|H_{k+l M, 1}\right|^{2}},
$$

and

$$
\alpha_{1}=\frac{E\left[\left|N_{k}\right|^{2}\right]}{E\left[\left|A_{k, 1}\right|^{2}\right]}
$$

(see [10]). This corresponds to a linear FDE, with a despreading operation implicit in the combination of the $K$ "replicas" of $A_{k, 1}$ (corresponding to a time-domain decimation procedure). For $K=1$ (i.e., without spreading), this corresponds to the conventional, linear FDE for CP-assisted SC modulations [10].

2) Iterative Receiver: The performance could be improved by replacing the FDE by an IB-DFE [12], [16]. In that case, for a given iteration, the frequency-domain samples at the output are given by

$$
\tilde{A}_{k, 1}=\sum_{l=0}^{K-1} Y_{k+l M} F_{k+l M, 1}-B_{k, 1} \hat{A}_{k, 1}
$$

where $\left\{F_{k, 1} ; k=0,1, \ldots, N-1\right\}$ and $\left\{B_{k, 1} ; k=\right.$ $0,1, \ldots, M-1\}$ denote the feedforward and the feedback coefficients, respectively. $\left\{\hat{A}_{k, 1} ; k=0,1, \ldots, M-1\right\}=$ DFT $\left\{\hat{a}_{n, 1} ; n=0,1, \ldots, M-1\right\}$, with $\left\{\hat{a}_{n, 1} ; n=0,1, \ldots, M-1\right\}$ denoting data estimates from the previous iteration (i.e., the hard decisions of the samples $\left\{\tilde{a}_{n, 1} ; n=0,1, \ldots, M-1\right\}$ from the previous iteration). The coefficients $F_{k, 1}$ and $B_{k, 1}$ are chosen so as to maximize the overall SNR (Signal-to-Noise Ratio) in the samples $\tilde{a}_{n, 1}$. Since this can be regarded as an IB-DFE receiver with $K$-order space diversity, from [16] it is 
clear that the optimum feedforward and feedback coefficients are given by

$$
B_{k, 1}=\rho_{1}\left(\sum_{l=0}^{K-1} F_{k+l M, 1} H_{k+l M, 1}-\gamma_{1}\right)
$$

and

$$
F_{k+l M, 1}=\frac{H_{k+l M, 1}^{*}}{\alpha_{1}+\left(1-\rho_{1}^{2}\right) \sum_{l=0}^{K-1}\left|H_{k+l M, 1}\right|^{2}},
$$

respectively, where

$$
\gamma_{1}=\frac{1}{M} \sum_{l=0}^{K-1} \sum_{k=0}^{M-1} F_{k+l M, 1} H_{k+l M, 1} .
$$

The coefficient $\rho_{1}$, which can be regarded as the blockwise reliability of the decisions used in the feedback loop (from the previous iteration), is given by

$$
\rho_{1}=\frac{E\left[\hat{A}_{k, 1} A_{k, 1}^{*}\right]}{E\left[\left|A_{k, 1}\right|^{2}\right]}=\frac{E\left[\hat{a}_{n, 1} a_{n, 1}^{*}\right]}{E\left[\left|a_{n, 1}\right|^{2}\right]} .
$$

This correlation coefficient $\rho_{1}$ could be computed as described in [16]. For the first iteration we do not have any information about $A_{k, 1}$ and the correlation coefficient is zero, corresponding to the linear FDE receiver. Once again, for $K=1$, this reduces to the IB-DFE receiver for $\mathrm{CP}$-assisted $\mathrm{SC}$ modulations [16].

\section{B. Linear MUD Receiver}

Let us assume now that we have $P$ users. In that case, we can employ the $K$ replicas associated to a given frequency domain sample $A_{k, p}$ to separate $P \leq K$ users. Therefore, the detection of the $p$ th user could be made based on $\left\{\tilde{a}_{n, p} ; n=\right.$ $0,1, \ldots, M-1\}=\operatorname{DFT}\left\{\tilde{A}_{k, p} ; k=0,1, \ldots, M-1\right\}$, where

$$
\tilde{A}_{k, p}=\sum_{l=0}^{K-1} F_{k+l M, p} Y_{k+l M}
$$

For each $k(k=0,1, \ldots, M-1)$, the $K$ coefficients $F_{k+l M, p}$, $l=0,1, \ldots, K-1$, are obtained by solving the following system of $K$ equations:

$$
\begin{array}{r}
H_{k+l M, p}^{*} \sum_{l^{\prime}=0}^{K-1} F_{k+l^{\prime} M, p} H_{k+l^{\prime} M, p}+ \\
+\sum_{p^{\prime} \neq p} H_{k+l M, p^{\prime}}^{*} \sum_{l^{\prime}=1}^{K-1} F_{k+l^{\prime} M, p} H_{k+l^{\prime} M, p^{\prime}}+ \\
+\alpha_{p} F_{k+l M, p}=H_{k+l M, p}^{*}, \quad l=0,1, \ldots, K-1,
\end{array}
$$

with $\alpha_{p}$ defined as in (8), i.e.,

$$
\alpha_{p}=\frac{E\left[\left|N_{k}\right|^{2}\right]}{E\left[\left|A_{k, p}\right|^{2}\right]} .
$$

IV. Iterative MUD Receiver with Interference Cancelation

\section{A. Receiver Structure}

We consider an iterative frequency-domain MUD receiver that combines IB-DFE principles with MAI cancelation. Each iteration consists of $P$ detection stages, one for each user. When detecting a given user, the MAI from previously detected users is canceled, as well as the residual ISI associated to that user. These MAI and residual ISI cancelations take into account the reliability of each of the previously detected users.

For a given iteration, the detection of the $p$ th user employs the structure depicted in fig. 1, where we have a feedforward filter, followed by a decimation procedure and $P$ feedback filters (one for each user). The feedforward filter is designed to minimize both the ISI and the MAI that cannot be canceled by the feedback filters, due to decision errors in the previous detection steps. After an IDFT operation, the corresponding time-domain outputs are passed through a hard-decision device so as to provide an estimate of the data block transmitted by the $p$ th user. For the case where we do not have any information about the users' data blocks, the receiver reduces to the linear frequency-domain MUD.

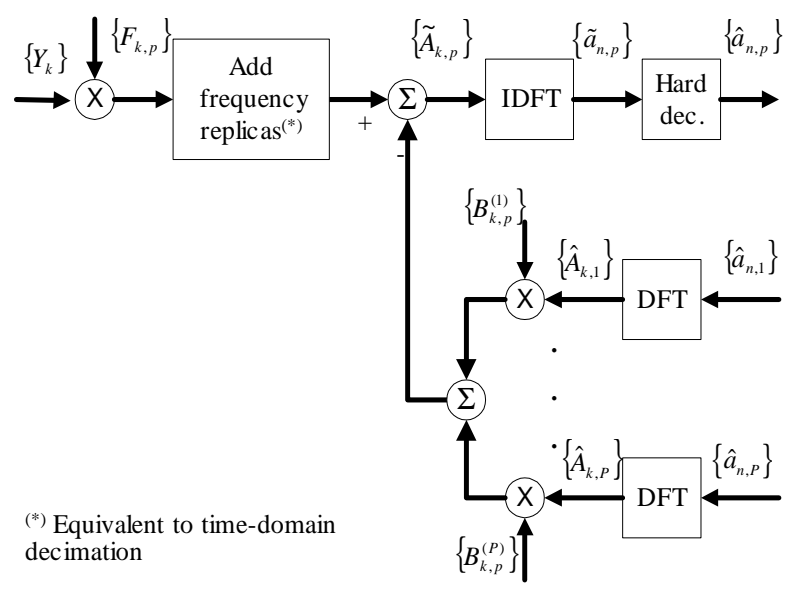

Fig. 1. Detection of the $p$ th user.

It is assumed that the users are ordered in descending order of their power. When detecting a given user, we cancel the MAI from all users (employing the most updated version of it), as well as the residual ISI for the user that is being detected.

\section{B. Derivation of the Receiver Coefficients}

For each iteration, the frequency-domain samples associated with the $p$ th user at the detector output are given by

$$
\begin{aligned}
& \tilde{A}_{k, p}=\sum_{l=0}^{K-1} F_{k+l M, p} Y_{k+l M}-\sum_{p^{\prime}=1}^{P} B_{k, p}^{\left(p^{\prime}\right)} \hat{A}_{k, p^{\prime}}= \\
= & \sum_{l=0}^{K-1} F_{k+l M, p} Y_{k+l M}-B_{k, p}^{(p)} \hat{A}_{k, p}-\sum_{p^{\prime} \neq p} B_{k, p}^{\left(p^{\prime}\right)} \hat{A}_{k, p^{\prime}}
\end{aligned}
$$

where $F_{k, p}(k=0,1, \ldots, N-1)$ denote the feedforward coefficients and $B_{k, p}^{\left(p^{\prime}\right)}(k=0,1, \ldots, M-1 ; p=1,2, \ldots, P)$ 
denote the feedback coefficients. The coefficients $\left\{B_{k, p}^{(p)} ; k=\right.$ $0,1, \ldots, M-1\}$ are used for residual ISI cancelation and the coefficients $\left\{B_{k, p}^{\left(p^{\prime}\right)} ; k=0,1, \ldots, M-1\right\}\left(p^{\prime} \neq p\right)$ are used for MAI cancelation. The block $\left\{\hat{A}_{k, p^{\prime}} ; k=0,1, \ldots, M-1\right\}$ is the DFT of the block $\left\{\hat{a}_{n, p^{\prime}} ; n=0,1, \ldots, M-1\right\}$, where the time-domain samples $\hat{a}_{n, p^{\prime}}, n=0,1, \ldots, M-1$, are the latest estimates for the $p^{\prime}$ th user transmitted symbols, i.e., the hard-decisions associated with the block of time-domain samples $\left\{\tilde{a}_{n, p^{\prime}} ; n=0,1, \ldots, M-1\right\}=\operatorname{IDFT}\left\{\tilde{A}_{k, p^{\prime}} ; k=\right.$ $0,1, \ldots, M-1\}$. For the $i$ th iteration $\hat{a}_{n, p^{\prime}}$ is associated with the $i$ th iteration for $p^{\prime}<p$ and with the $(i-1)$ th iteration for $p^{\prime} \geq p$ (in the first iteration, we do not have any information for $p^{\prime} \geq p$ and $\hat{a}_{n, p^{\prime}}=0$ ).

Due to decision errors, we have $\hat{a}_{n, p} \neq a_{n, p}$ for some symbols. Consequently, $\hat{A}_{k, p} \neq A_{k, p}$. For the computation of the receiver coefficients, it is assumed that

$$
\hat{A}_{k, p}=\rho_{p} A_{k, p}+\Delta_{k, p}
$$

where $E\left[\Delta_{k, p}\right] \approx 0, E\left[\Delta_{k, p} A_{k^{\prime}, p}\right] \approx 0$, regardless of $k$ and $k^{\prime}$, and $E\left[\left|\Delta_{k, p}\right|^{2}\right]=\left(1-\rho_{p}^{2}\right) E\left[\left|A_{k, p}\right|^{2}\right]$. The correlation coefficient $\rho_{p}$ is defined as in (13), i.e.,

$$
\rho_{p}=\frac{E\left[\hat{A}_{k, p} A_{k, p}^{*}\right]}{E\left[\left|A_{k, p}\right|^{2}\right]}=\frac{E\left[\hat{a}_{n, p} a_{n, p}^{*}\right]}{E\left[\left|a_{n, p}\right|^{2}\right]} .
$$

By combining (5), (17) and (18), we obtain

$$
\begin{aligned}
& \tilde{A}_{k, p}=\sum_{l=0}^{K-1} F_{k+l M, p}\left(\sum_{p^{\prime}=1}^{P} H_{k+l M, p^{\prime}} A_{k, p^{\prime}}+N_{k+l M}\right)- \\
& -B_{k, p}^{(p)}\left(\rho_{p} A_{k, p}+\Delta_{k, p}\right)-\sum_{p^{\prime} \neq p} B_{k, p}^{\left(p^{\prime}\right)}\left(\rho_{p^{\prime}} A_{k, p^{\prime}}+\Delta_{k, p^{\prime}}\right)= \\
& =\underbrace{\gamma_{p} A_{k, p}}_{\text {Useful signal }}+ \\
& +\underbrace{\left(\sum_{l=0}^{K-1} F_{k+l M, p} H_{k+l M, p}-\gamma_{p}-\rho_{p} B_{k, p}^{(p)}\right) A_{k, p}}+ \\
& \text { Residual ISI } \\
& +\underbrace{\sum_{p^{\prime} \neq p}\left(\sum_{l=0}^{K-1} F_{k+l M, p} H_{k+l M, p^{\prime}}-\rho_{p^{\prime}} B_{k, p}^{\left(p^{\prime}\right)}\right) A_{k, p^{\prime}}}_{\text {Residul MAI }}- \\
& \text { Residual MAI } \\
& \text { - } \underbrace{\sum_{p^{\prime}=1}^{P} B_{k, p}^{\left(p^{\prime}\right)} \Delta_{k, p^{\prime}}}_{\text {,Nois }}+\underbrace{\sum_{l=0}^{K-1} F_{k+l M, p} N_{k+l M}}_{\text {Channel noise }} \text {, }
\end{aligned}
$$

with

$$
\gamma_{p}=\frac{1}{M} \sum_{k=0}^{M-1} \sum_{l=0}^{K-1} F_{k+l M, p} H_{k+l M, p}
$$

The forward and backward coefficients, $\left\{F_{k, p} ; k=\right.$ $0,1, \ldots, N-1\}$ and $\left\{B_{k, p}^{\left(p^{\prime}\right)} ; k=0,1, \ldots, M-1\right\}, p^{\prime}=$ $1,2, \ldots, P$, respectively, are chosen so as to maximize the "signal-to-noise plus interference ratio" for the $p$ th user. It can be shown that the optimum feedforward coefficients are the solution of the system of $K$ equations:

$$
\begin{array}{r}
\left(1-\rho_{p}^{2}\right) H_{k+l M, p}^{*} \sum_{l^{\prime}=0}^{K-1} F_{k+l^{\prime} M, p} H_{k+l^{\prime} M, p}+ \\
+\sum_{p^{\prime} \neq p}\left(1-\rho_{p^{\prime}}^{2}\right) H_{k+l M, p^{\prime}}^{*} \sum_{l^{\prime}=0}^{K-1} F_{k+l^{\prime} M, p} H_{k+l^{\prime} M, p^{\prime}}+ \\
+\alpha_{p} F_{k+l M, p}= \\
=H_{k+l M, p}^{*}, \quad l=0,1, \ldots, K-1 .
\end{array}
$$

and the optimum feedback coefficients are given by

$$
B_{k, p}^{\left(p^{\prime}\right)}=\rho_{p^{\prime}}\left(\sum_{l^{\prime}=0}^{K-1} F_{k+l^{\prime} M, p} H_{k+l^{\prime} M, p^{\prime}}-\gamma_{p} \delta_{p, p^{\prime}}\right),
$$

$p^{\prime}=1,2, \ldots, P,\left(\delta_{p, p^{\prime}}=1\right.$ if $p=p^{\prime}$ and 0 otherwise $)$. It should be noted that the feedforward and feedback coefficients obtained this way are closely related to the optimum coefficients for the iterative layered space-time receivers proposed in [17]. This results from the fact that frequency-domain receivers with ISI and interference cancelation are considered in both cases (in our case we have MAI and in [17] there is "interlayer" interference); moreover, in both cases, the user/layer separation is based on multiple replicas of each frequencydomain sample (in our case this multiplicity is inherent to the transmitted signals, while, in [17], it results from employing multiple antennas at the receiver).

If we do not have data estimates for the different users $\rho_{p^{\prime}}=$ $0, p^{\prime}=1,2, \ldots, P$ and the feedback coefficients are zero. Therefore, (22) reduces to (15), i.e., we have the linear MUD described in the previous section.

It can also be shown that the solution of the system of (22) can be written in the form

$$
F_{k+l M, p}=\sum_{p^{\prime}=1}^{P} H_{k+l M, p^{\prime}}^{*} I_{k, p}^{\left(p^{\prime}\right)}
$$

( $k=0,1, \ldots, M-1 ; l=0,1, \ldots, K-1)$, with the set of coefficients $\left\{I_{k}^{\left(p^{\prime}\right)} ; p^{\prime}=1,2, \ldots, P\right\}$ satisfying the set of $P$ equations

$$
\sum_{p^{\prime \prime}=1}^{P} I_{k, p}^{\left(p^{\prime \prime}\right)}
$$

$$
\begin{array}{r}
\left(\left(1-\rho_{p^{\prime}}^{2}\right) \sum_{l^{\prime}=0}^{K-1} H_{k+l^{\prime} M, p^{\prime \prime}}^{*} H_{k+l^{\prime} M, p^{\prime}}+\alpha_{p} \delta_{p^{\prime}, p^{\prime \prime}}\right)= \\
=\delta_{p, p^{\prime}}, \quad p^{\prime}=1,2, \ldots, P .
\end{array}
$$

The computation of the feedforward coefficients from (24) is simpler than the direct computation, from (22), especially when $P<K$. 


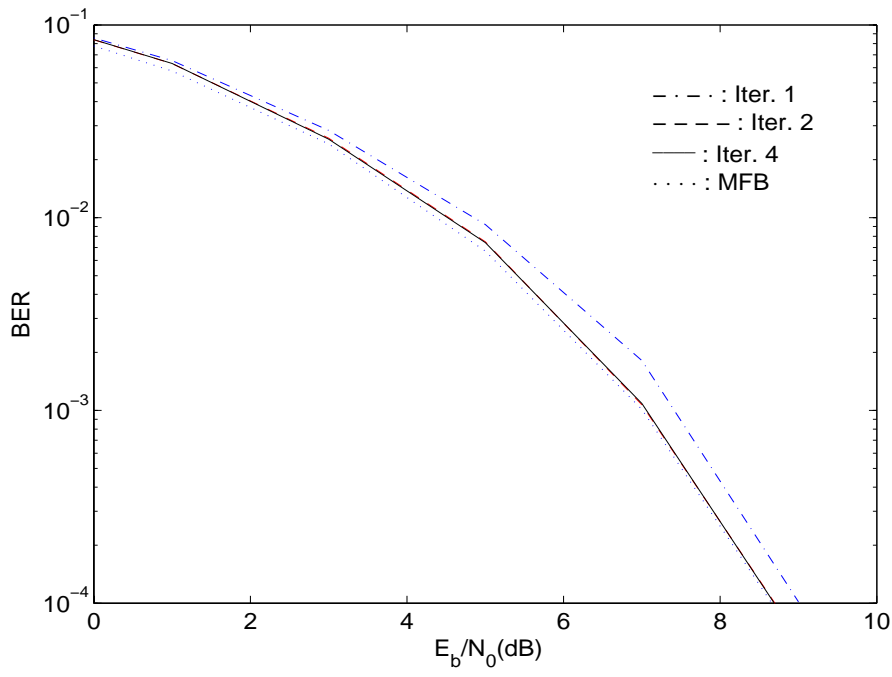

Fig. 2. BER for a single-user scenario and a receiver with 1, 2 or 4 iterations.

\section{Performance Results}

In this section, we present a set of performance results concerning the proposed MUD receiver. We consider the uplink transmission within a CP-assisted DS-CDMA system with spreading factor $K=8$ and $M=64$ data symbols for each user, corresponding to blocks with length $N=K M=512$, plus an appropriate cyclic extension. QPSK constellations, with Gray mapping, are employed. We consider severely timedispersive channels and uncoded BER performances under perfect synchronization and channel estimation conditions.

Let us first assume that the signals associated to all users have the same average power at the receiver (i.e., the BS), which corresponds to a scenario where an "ideal average power control" is implemented. Figs. 2 to 4 show the impact of the number of iterations on the BER for each user. For the sake of comparisons, we also include the corresponding MFB performance (Matched Filter Bound). From these figures, we can observe that our iterative receiver structure allows a significant improvement on the BER performance, especially when the number of users is high. For a given iteration, the users that are detected first face stronger interference levels and have worse BER. This is especially important at the first iteration and when the number of users is high. After four iterations the performances are already similar for all users, and very close to the MFB, even for a fully loaded scenario (i.e., when $P=K=8$ ).

Let us consider now a scenario where the signals associated to different users have different average powers at the receiver. We will consider two classes of users, denoted by $C_{L}$ and $C_{H}$, with different average powers at the receiver. The performance results presented in fig. 5 concern the case where the average power of $C_{H}$ users is $6 \mathrm{~dB}$ above the average power of $C_{L}$ users; for fig. 6 , it is assumed that average power of $C_{H}$ users is $10 \mathrm{~dB}$ above the average power of $C_{L}$ users. In both

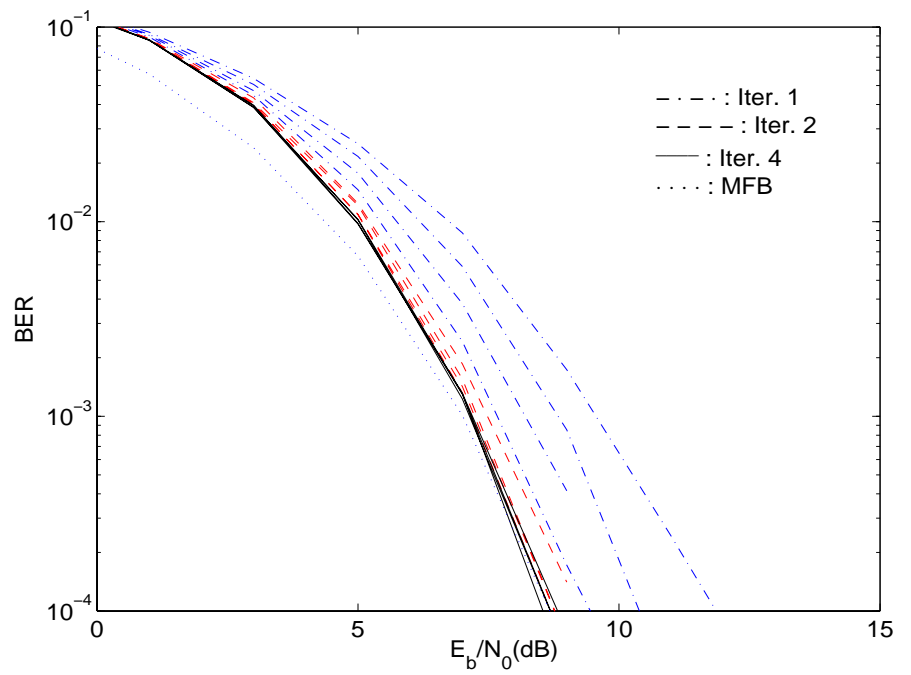

Fig. 3. BER for each user, when $P=4$ and a receiver with 1,2 or 4 iterations (for a given iteration, the users that are detected later have better BER).

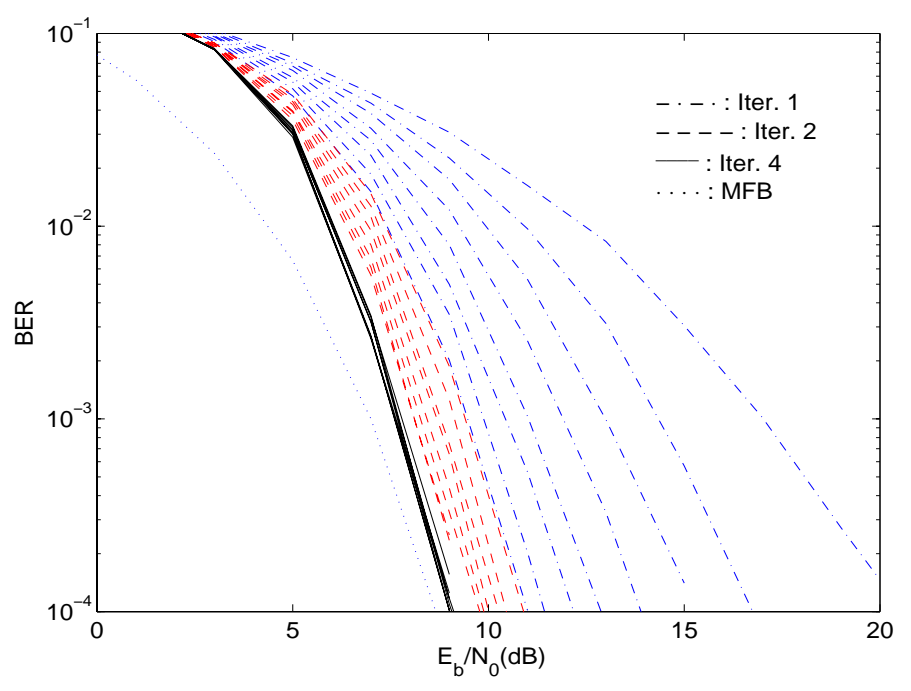

Fig. 4. As in fig. 3, but for $P=8$ users.

cases, we have a fully loaded scenario, with $4 C_{L}$ users and $4 C_{H}$ users. Clearly, the $C_{L}$ users face strong interference conditions. Once again, the proposed iterative receiver allows significant performance gains. In both cases, the performance of low power users asymptotically approaches the MFB when we increase the number of iterations; however, for high power users, the BER at $10^{-4}$ is still between 1 or $2 \mathrm{~dB}$ from the MFB. This can be explained from the fact that the BER is much lower for high-power users, allowing an almost perfect interference cancelation of their effects on low-power users; therefore, the corresponding performances can be very close to the MFB. The higher BERs for the low-power users preclude an appropriate interference cancelation when we detect highpower users. 


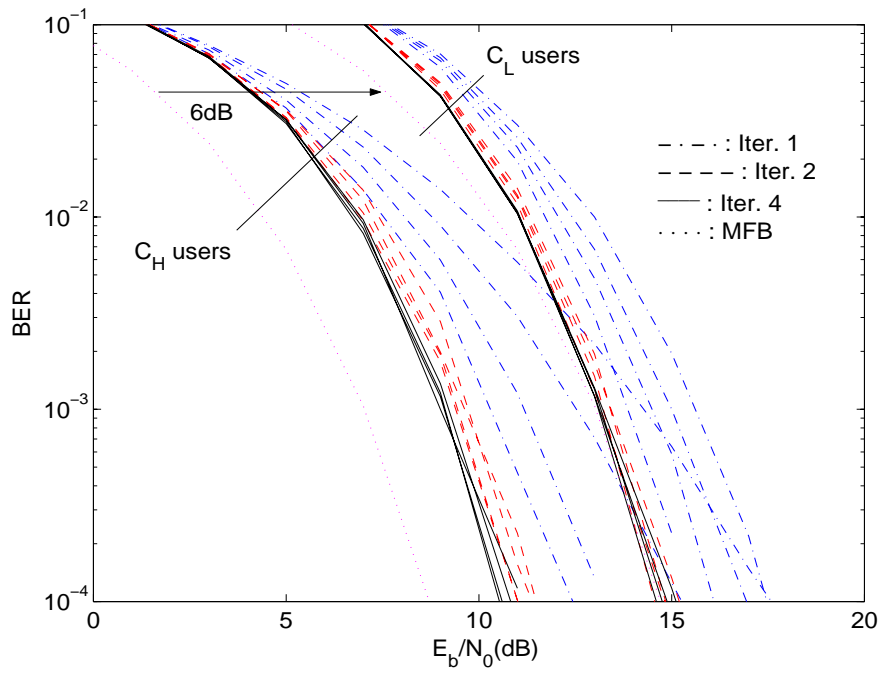

Fig. 5. BER performances as a function of the $E_{b} / N_{0}$ of $C_{H}$ users (average power of $C_{H}$ users $6 \mathrm{~dB}$ above the average power of $C_{L}$ users).

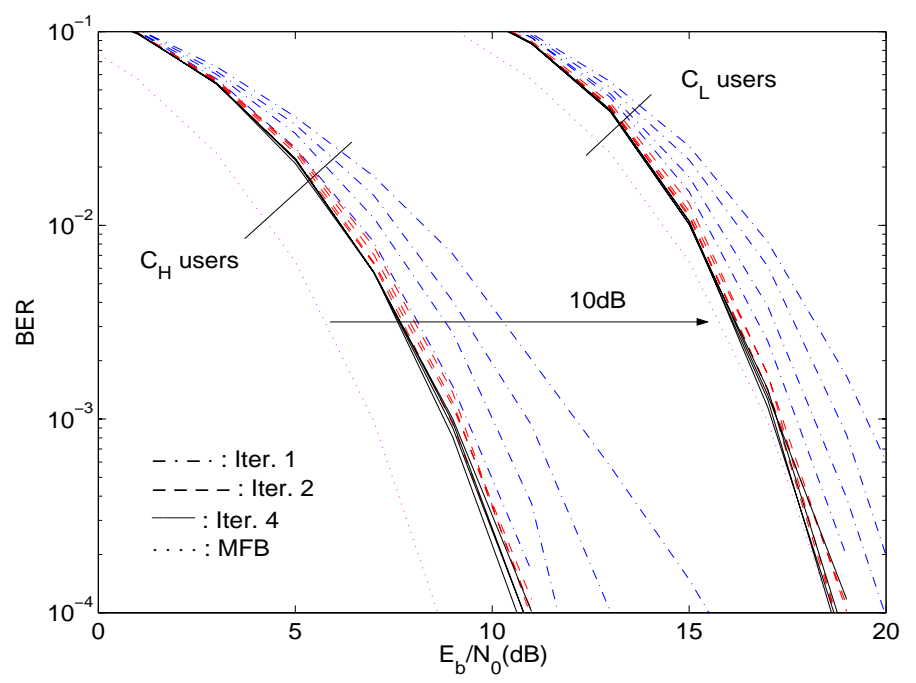

Fig. 6. BER performances as a function of the $E_{b} / N_{0}$ of $C_{H}$ users (average power of $C_{H}$ users $10 \mathrm{~dB}$ above the average power of $C_{L}$ users).

\section{Conclusions}

An iterative frequency-domain MUD scheme was proposed for the uplink of CP-assisted DS-CDMA systems that combines IB-DFE principles with MAI cancelation.

Our performance results show that the proposed receiver is appropriate for DS-CDMA wireless systems, with performances very close to the single-user MFB after just a few iterations, even for fully loaded systems, in severe timedispersive channels and/or in the presence of strong interfering signals.

\section{References}

[1] A. Viterbi, CDMA: Principles of SS Communication, Addison Wesley, 1995.
[2] A. Gusmão, R. Dinis, J. Conceição, and N. Esteves, "Comparison of Two Modulation Choices for Broadband Wireless Communications", IEEE VTC'00 (Spring), Tokyo, Japan, May 2000.

[3] D.Falconer, S.Ariyavisitakul, A.Benyamin-Seeyar and B.Eidson, "Frequency Domain Equalization for Single-Carrier Broadband Wireless Systems", IEEE Comm. Mag., Vol. 4, No. 4, pp. 58-66, April 2002.

[4] R. Dinis, D. Falconer, C. Lam and M. Sabbaghian, "A Multiple Access Scheme for the Uplink of Broadband Wireless Systems", in IEEE GLOBECOM'04, Dec. 2004.

[5] C. Chang and K. Chen, "Frequency-Domain Approach to Multiuser Detection in DS-CDMA Communications", in IEEE Comm. Letters, Vol. 4, No 11, pp. 331-333, Nov. 2000.

[6] D. Galda and H. Rohling, "A Low-Complexity Transmitter Structure for OFDM-FDMA Uplink Systems", in Proc. VTC'02(Spring), pp. 1737-1741, May 2002.

[7] P. Xia, S. Zhou and G. Giannakis, "Bandwidth- and PowerEfficient Multi-Carrier Multiple Access", in Proc. WCNC, pp. 207-211, March 2003.

[8] S. Barbarossa and F. Cerquetti, "Simple Space-Time Coded SSCDMA Systems Capable of Perfect MUI/ISI Elimination", IEEE Comm. Letters, Vol. 5, No. 12, pp. 471-473, Dec. 2001.

[9] K. Baum, T. Thomas, F. Vook, V. Nangia, "Cyclic-Prefix CDMA: An Improved Transmission Method for Broadband DS-CDMA Cellular Systems", IEEE WCNC, pp. 183-188, 2002.

[10] A.Gusmão, R.Dinis and N.Esteves, "On Frequency-domain Equalization and Diversity Combining for Broadband Wireless Communications", IEEE Trans. on Comm., Vol. 51, No. 7, pp. 1029-1033, July 2003.

[11] P. Silva and R. Dinis, "An Iterative Frequency-Domain Decision Feedback Receiver for CDMA Systems”, , ISWCS'04, Sep. 2004.

[12] N. Benvenuto and S. Tomasin, "Block Iterative DFE for Single Carrier Modulation", IEE Elec. Let., Vol. 39, No. 19, pp. 11441145, Sep. 2002.

[13] M. Tüchler, R. Koetter and A. Singer, "Turbo Equalization: Principles and New Results", IEEE Trans. on Comm., Vol. 50, May 2002.

[14] T. Ojamperä and R. Prasad, Wideband CDMA for Third Generation Mobile Communications, Artech House Publ., 1998.

[15] W. Gardner, "Exploitation of Spectral Redundancy in Cyclostationary Signals", IEEE Signal Processing Mag., Vol. 9, Aug. 1991.

[16] R. Dinis, A. Gusmão, and N. Esteves, "On Broadband Block Transmission over Strongly Frequency-Selective Fading Channels”, Proc. Wireless 2003, Calgary, Canada, July 2003.

[17] R. Dinis, R. Kalbasi, D. Falconer and A. Banihashemi, "Iterative Layered Space-Time Receivers for Single-Carrier Transmission over Severe Time-Dispersive Channels", IEEE Comm. Letters, Vol. 8, No. 9, pp. 579-581, Sep. 2004. 